\title{
Fibrossarcoma perirrenal em um gato acometido por Dioctophyme renale em rim
}

\section{esquerdo}

\author{
Perirenal Fibrosarcoma in a cat affected by Dioctophyme renale in a left kidney \\ Fibrossarcoma perirrenal en un gato afectado por Dioctophyme renale en un riñón izquierdo
}

Recebido: 02/05/2021 | Revisado: 10/05/2021 | Aceito: 22/05/2021 | Publicado: 08/07/2021

\author{
Aline Xavier Fialho Galiza \\ ORCID: https://orcid.org/0000-0002-1139-7117 \\ Universidade Federal de Pelotas, Brasil \\ E-mail: aline.xavfialho@hotmail.com \\ Pâmela Caye \\ ORCID: https://orcid.org/0000-0002-6456-8100 \\ Universidade Federal de Santa Maria, Brasil \\ E-mail: pamiscaye@gmail.com \\ Thomas Normanton Guim \\ ORCID: https://orcid.org/0000-0001-8278-1397 \\ Universidade Federal de Pelotas, Brasil \\ E-mail: thomasguim@ @otmail.com \\ Josaine Cristina da Silva Rappeti \\ ORCID: https://orcid.org/0000-0002-8822-0387 \\ Universidade Federal de Pelotas, Brasil \\ E-mail: josainerappeti@yahoo.com.br \\ Luísa Grecco Correa \\ ORCID: https://orcid.org/0000-0003-1355-2907 \\ Universidade Federal de Pelotas, Brasil \\ E-mail: luisagcorrea@gmail.com \\ Luísa Mariano Cerqueira da Silva \\ ORCID: https://orcid.org/0000-0001-6158-4204 \\ Universidade Federal de Pelotas, Brasil \\ E-mail: luisamarianovet@yahoo.com.br \\ Jéssica Paola Salame \\ ORCID: https://orcid.org/0000-0001-8470-9292 \\ Universidade Federal de Pelotas, Brasi \\ E-mail: dassi.jessica@hotmail.com \\ Márcia de Oliveira Nobre \\ ORCID: https://orcid.org/0000-0003-3284-9167 \\ Universidade Federal de Pelotas, Brasi \\ E-mail: marciaonobre@gmail.com \\ Katiellen Ribeiro Neves \\ ORCID: https://orcid.org/0000-0002-0180-222X \\ Universidade Federal de Pelotas, Brasil \\ E-mail: katiellenribeirodasneves@hotmail.com \\ Mariana Cristina Hoeppner Rondelli \\ ORCID: https://orcid.org/0000-0001-5385-8228 \\ Universidade Federal de Pelotas, Brasil \\ E-mail: marianarondelli@gmail.com \\ Cristina Gevehr Fernandes \\ ORCID: https://orcid.org/0000-0002-0729-2099 \\ Universidade Federal de Pelotas, Brasil \\ E-mail: crisgevf@yahoo.com.br \\ Fabiane Borelli Grecco \\ ORCID: https://orcid.org/0000-0002-3996-315X \\ Universidade Federal de Pelotas, Brasil \\ E-mail: fabianegrecco18@gmail.com
}

\section{Resumo}

O objetivo deste trabalho foi relatar a ocorrência de parasitismo por Dioctophyme renale, em rim esquerdo de um felino que também apresentou fibrossarcoma perirrenal esquerdo. Foi atendida no Hospital de clínicas veterinárias da UFPEL uma gata de 10 anos com queixa de dor abdominal e prostração. Exames de imagem sugeriram neoplasma em região perirrenal esquerda. $\mathrm{O}$ animal foi submetido a laparotomia, onde observou-se massa tumoral aderida ao rim esquerdo, motivo que levou a realização d nefrectomia A massa tumoral e o rim esquerdo foram encaminhados para 
estudo histopatológico. No exame macroscópico o rim acometido apresentava atrofia do parênquima, cápsula espessada e aspecto cístico com presença de líquido amarelado contendo estruturas degeneradas identificadas como Dioctophyme renale. A massa tumoral era esbranquiçada, firme e bem vascularizada. Histologicamente a massa era constituída por células neoplásicas pleomórficas, sarcomatosas que podiam estar arranjadas em feixes ou ninhos. A coloração de Tricrômicro de Masson evidenciou fibras colágenas e estroma de tecido conjuntivo fibroso permitindo o diagnóstico de fibrossarcoma. Neste relato, três fatos incomuns foram discutidos: a) ocorrência de Dioctophyme renale em felinos b) envolvimento do rim esquerdo c) fibrossarcoma perirrenal com possibilidade de relação ao parasitismo por Dioctophyme renale.

Palavras-chave: Neoplasias; Felinos; Sarcomas.

\begin{abstract}
The objective of this study was to report the occurrence of Dioctophyme renale in the left kidney of feline along with the presence, and the occurrence of left perirenal fibrosarcoma. It was attended in hospital veterinary of the UFPel at a 10 -year-old cat complaining of abdominal pain and prostration was treated. Imaging studies suggested neoplasms in the left perirenal region. The animal underwent laparotomy where intense adhesion of a tumor mass to the kidney was observed, which led to nephrectomy of the left kidney. Tumor mass and left kidney were referred for histopathological study. On macroscopic examination, the affected kidney had parenchymal atrophy and the organ capsule was thickened and cystic aspect was seen with a yellowish liquid content and structures similar to a degenerate Dioctophyme renale. The tumor mass was whitish, firm and well vascularized. Histologically, pleomorphic, sarcomatous neoplastic cells and fibrous connective tissue stroma were observed that could be arranged in bundles or nests. The Masson's Trichrome staing showed collagen fibers and fibrous connective tissue stroma allowing the diagnosis of fibrosarcoma. In this report, three unusual facts were discussed: a) occurrence of Dioctophyme renale in felines b) left kidney involvement. c) perirenal fibrosarcoma with the suggestion of some involvement with Dioctophyme renale parasitism.
\end{abstract}

Keywords: Neoplasms; Felines; Sarcoma.

\title{
Resumen
}

El objetivo de este estudio fue informar la ocurrencia de Dioctophyme renale, en el riñón izquierdo de un felino que también lo tenía, y la ocurrencia de fibrosarcoma perirrenal izquierdo. Un gato de 10 años con dolor abdominal y próteses fue tratado en el hospital de la clínica veterinaria UFPel. Los exámenes de imagen sugerían una neoplasia en la región perirrenal izquierda. El animal fue sometido a laparotomía donde se observó una intensa adhesión de una masa tumoral al riñón, que condujo a la anfrectomía del riñón izquierdo y la masa tumoral y el riñón izquierdo se enviaron para su estudio histopatológico. En el examen macroscópico, el riñón afectado presentaba atrofia del parénquima y la cápsula del órgano estaba engrossada, presentaba un aspecto quístico con un líquido amarillento que contenía estructuras similares al Dioctophyme renale degenerado. La masa tumoral era blanquecina, firme y bien vascularizada. Histológicamente se observaron células neoplásicas pleomórficas y sarcomatosas que podían disponerse em haces o nidos. La tinción con Tricrómico de Masson mostró fibras de colágeno y estroma de tejido conectivo fibroso, lo que permitió el diagnóstico de fibrosarcoma. En este informe se discuten tres hechos inusuales: a) aparición de Dioctophyme renale en felinos b) afectación del riñón izquierdo c) el fibrosarcoma perirrenal como sugerencia de alguna implicación con el parasitismo de Dioctophyme renale.

Palabras clave: Neoplasias; Felinos; Sarcoma.

\section{Introdução}

O Dioctophyme renale é um parasito nematódeo, popularmente conhecido como verme gigante renal, que parasita diferentes espécies de animais, com maior frequência os cães e outros animais domésticos e selvagens, mas atípicos em gatos (Pedrassani et al., 2014). Em felinos poucos casos foram notificados no Brasil, sendo 3 casos em Pelotas/RS (Rappeti et al. 2016; Silva et al., 2017). Também, foram diagnosticados dois casos de dioctofimatose em gatos, os quais apresentaram sintomas inespecíficos (Verocai et al., 2009; Pedrassani et al., 2014).

Os animais parasitados normalmente são assintomáticos (Souza et al., 2019), portanto o diagnóstico de Dioctofimatose ocorre através de achados ocasionais dos helmintos em necropsias, procedimentos cirúrgicos, exames de ultrassom e presença de ovos ou nematódeos na urina (Perera et al., 2017). O nematódeo se instala, comumente, no rim direito de seus hospedeiros definitivos, alimentando-se do seu parênquima. O parasito libera enzimas esofágicas, causando necrose nos locais atingidos, o que possibilita a penetração e destruição renal, resultando em danos irreparáveis ao órgão (Sapin et al., 2017). 
Fibrossarcomas são neoplasias malignas que se originam de fibroblastos (Meuten, 2017). Felinos apresentam maior probabilidade no desenvolvimento de sarcomas, inclusive com a formação dessas massas em reações pós-vacinais (Ferreira et al., 2016). Alguns felinos podem ser geneticamente predispostos a desenvolver essa neoplasia na presença de processos inflamatórios ou regenerativos, quando as células perdem suas propriedades antiproliferativas e tornam-se malignas (Perlmann et al., 2011).

Os objetivos deste trabalho foram trazer à discussão dois fatos incomuns: A ocorrência de Dioctophyme renale em felinos, localizado no rim esquerdo e fibrossarcoma perirrenal com sugestão de envolvimento com o parasitismo como desencadeador de oncogênese.

\section{Metodologia}

Foi atendida no Hospital de Clínicas Veterinárias da Universidade Federal de Pelotas (HCV - UFPel) uma gata de 10 anos, não castrada, prostrada e inapetente. A paciente foi internada e recebeu tratamento de suporte, e submetida a exames complementares (ultrassonografia e hematológicos). $\mathrm{O}$ animal foi encaminhado para laparotomia e nefrectomia esquerda. O rim esquerdo e fragmento da massa perirrenal foram enviados para exame anatomopatológico ao Serviço de Oncologia Veterinário da UFPel (SOVET/UFPel) onde foram processados, clivados e corados rotineiramente.

\section{Resultados e Discussão}

Ao exame ultrassonográfico da região abdominal observou-se presença de massa de aspecto tumoral em topografia renal esquerda. O hemograma não apresentava alterações e o leucograma apresentava leucocitose com desvio a esquerda e trombocitopenia; o exame bioquímico apresentava aumento na concentração de uréia e fosfatase alcalina abaixo dos valores de referências. Após a estabilização, a paciente foi encaminhada ao centro cirúrgico e submetida à celiotomia exploratória. Na inspeção da cavidade abdominal se constatou presença de líquido livre e observou-se o rim esquerdo com aspecto macroscópico alterado, globoso, esbranquiçado e firme. A área perirrenal esquerda estava envolta por tecido esbranquiçado e firme e incluía vasos renais, tecidos ósseos vertebrais e o peritônio local. Procedeu-se com nefrectomia esquerda e, remoção da massa.

A massa (Figura 1) estava aderida à cápsula renal esquerda e media 7,8x6,0x5,0 cm, firme ao corte e esbranquiçada. O rim esquerdo (Figura 2) se apresentava cístico, firme, esbranquiçado e com perda completa da arquitetura tecidual renal. Havia conteúdo líquido amarelado e exemplares de Dioctophyme renale em estado avançado de degeneração. Microscopicamente, o rim esquerdo apresentava hipertrofia e espessamento da cápsula renal com áreas de metaplasia óssea. A massa adjacente ao rim (Figura 3) era constituída por células neoplásicas pleomórficas, sarcomatosas e podiam estar arranjadas em feixes ou ninhos. Havia neovascularização acentuada e hipertrofia das células endoteliais vasculares. O diagnóstico do neoplasma foi fibrossarcoma perirrenal. 
Research, Society and Development, v. 10, n. 6, e50410615705, 2021

(CC BY 4.0) | ISSN 2525-3409 | DOI: http://dx.doi.org/10.33448/rsd-v10i6.15705

Figura 1. Massa aderida à cápsula renal esquerda, medindo 7,8x6,0x5,0 cm, firme ao corte e esbranquiçada.

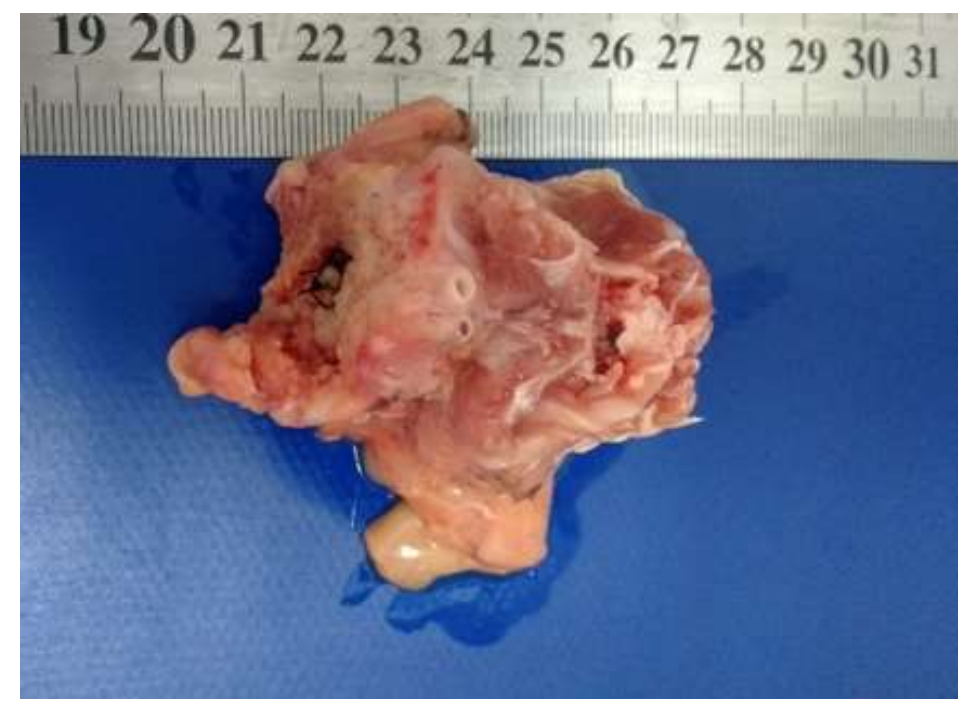

Fonte: Sovet (2018).

Figura 2. Rim esquerdo cístico, firme, esbranquiçado e com perda completa da arquitetura do tecido renal. Em seu interior, havia conteúdo líquido amarelado e exemplares de Dioctophyme renale em estado avançado de degeneração.

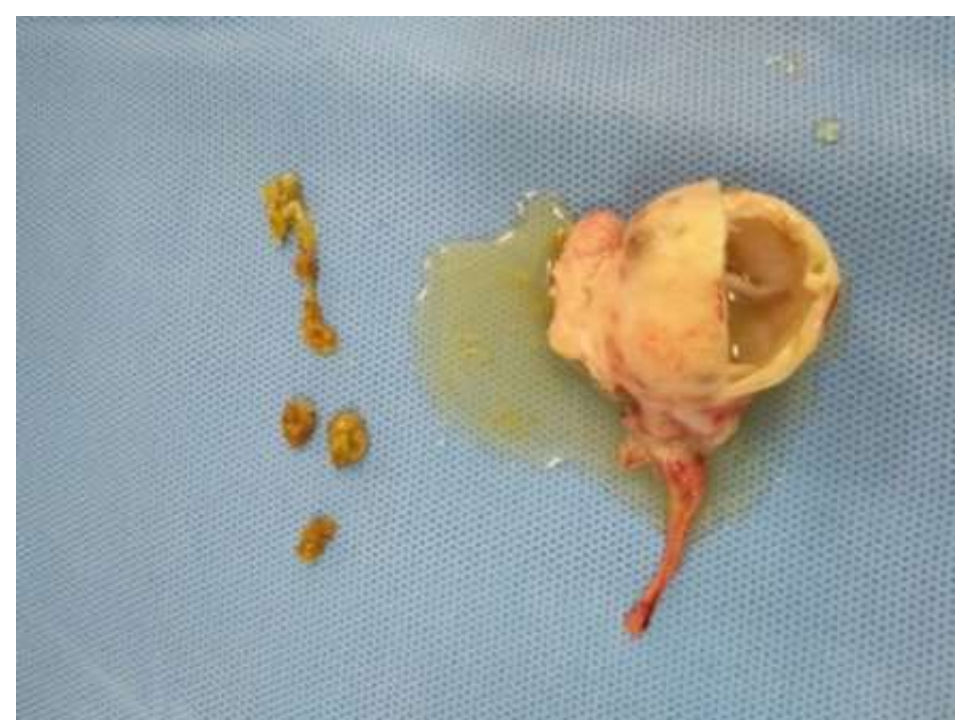

Fonte: Sovet (2018). 
Figura 3. Microscopia da massa aderida ao rim esquerdo de um felino. a) Metaplasia óssea em cápsula renal esquerda. b) e c) células pleomórficas e sarcomatosas. fibrossarcoma perirrenal esquerdo.

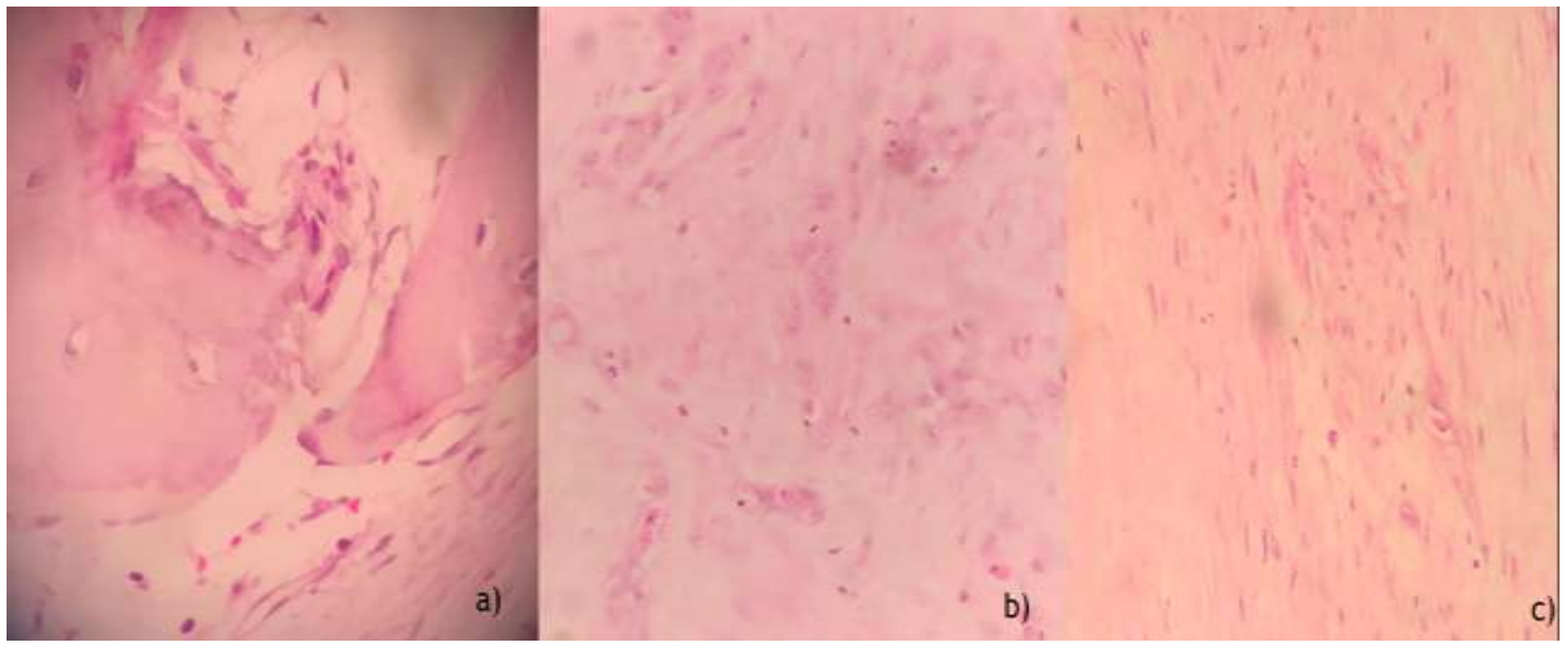

Fonte: Sovet (2018).

A dioctofimatose é considerada uma zoonose e apresenta distribuição mundial (Barriga, 2002; Norouzi et al., 2017). Os cães são os principais hospedeiros definitivos para Dioctophyme renale quando se trata de espécies de animais domésticos. No entanto, a parasitose em felinos embora rara foi relatada por Rappeti, et al. (2016), porém em todos esses casos o rim direito era o acometido.

O parasito tem predileção pelo rim direito, justificada pela proximidade deste com o duodeno, local de penetração da larva infectante após ingestão alimentar (Pereira et al., 2006). Eventualmente, Dioctophyme renale pode penetrar pela curvatura maior do estômago e migrar ao rim esquerdo ou cavidade abdominal, justificando o achado de parasitos ectópicos (Kommers et al., 1999; Zabott et al., 2012). Ao ser parasitado, o rim começa a sofrer destruição das camadas medular e cortical renais, principal característica macroscópica da parasitose, causadas pela ação das enzimas proteolíticas e lipolíticas esofágicas produzidas pelo parasito. Tal ação reduz o órgão a uma cápsula fibrosa e, a constante agressão na parede renal, pode levar ao desenvolvimento de metaplasia óssea (Sapin et al., 2017).

A metaplasia óssea que ocorre na cápsula renal de cães parasitados é uma alteração reversível de um tipo celular diferenciado (mesenquimal-conjuntivo), por outro tipo celular de mesma linhagem (óssea) (Sapin et al., 2017). Nessa substituição de tipo celular ocorre uma reprogramação de células precursoras que se diferenciam. As influências que desencadeiam a metaplasia, se persistentes, poderiam iniciar transformação maligna nas células metaplásicas (Robbins et al., 2015), além disso, uma das alterações frequentemente descritas nas lesões provocadas por Dioctophyme renale são a fibroplasia acentuada, hiperplasia e hipertrofia vascular (Sapin et al., 2017).

Fibrossarcomas podem se desenvolver como resultado de uma resposta inflamatória exacerbada em gatos. Características individuais herdadas também podem desempenhar um papel fundamental no desenvolvimento de tais neoplasmas (Nambiar et al., 2001). Acredita-se que células com predisposição neoplásica podem estar presentes no tecido antes da agressão de fatores físicos ou químicos externos, com a presença de um corpo estranho ou substância, que no organismo resulta em uma reação inflamatória, podendo levar ao surgimento de um sarcoma em sua vizinhança (Kirkpatrick et al., 2000; Moizhess, 2008). As neoplasias de tecidos moles podem surgir em qualquer localização no corpo, mas a pele e tecido subcutâneo mantêm-se os locais com maior incidência (Buracco, 2015).

Descrita por Peiffer, et al. (1998) a ocorrência de sarcomas intraoculares em gatos que sofreram trauma ou uveíte 
crônica, o que corrobora com a hipótese do papel inflamatório (Hakanson et al., 1990), a inflamação crônica causada por infecção parasitária também foi associada como causa do desenvolvimento de tumor em várias espécies animais incluindo canídeos, roedores e humanos, comprovando que o componente inflamatório é uma das chaves para a patogênese do sarcoma (Ranen et al., 2004). Portanto, sugere-se que a agressão persistente das enzimas esofágicas produzidas pelo parasito pode ter estimulado o desenvolvimento de um fibrossarcoma adjacente ao rim esquerdo. Ainda deve ser salientada a predisposição dos felinos a um maior desenvolvimento de sarcomas (Ferreira et al., 2016).

\section{Considerações Finais}

A análise histopatológica criteriosa de rins e/ou outros tecidos de animais acometidos por Dioctophyme renale é importante para contribuição dos estudos sobre o parasitismo e para avaliar uma possível correlação da dioctofimatose e ocorrência de neoplasmas.

\section{Referências}

Barriga, O. (2002). Las enfermidades parasitarias de los animales domésticos em la America Latina. Santiago: Editorial Germinal. p.247.

Buracco, P. (2015). Canine and feline soft tissue sarcomas. Apresentado e publicado no CD de Proceedings do XXIII Congresso Nacional APMVEAC: Oncologia, neurologia e patologia clínica, Lisboa, Portugal, 6-7 de Junho.

Ferreira, M. G. P. A., Filho, N. P. R., Pascoli, A. L. C. R., Storti, R. P., Oliveira, I. R. C., \& Di Nardi, A.B. (2016). Sarcoma de aplicação em felinos: aspectos clínicos, diagnóstico e terapia. Revista Investigação, 15(7), 29-36.

Hakanson, N., Shively, J. N., Reed, R. E., \& Meredith, R. E. (1990). Intraocular spindle cell sarcoma following ocular trauma in a cat: Case report and literature review. The Journal of the American Animal Hospital Association, 26, 63-66.

Kirkpatrick, C. J., Alves, A., Kohler, H., Kriegsmann, J., Bittinger, F., Otto, M., Williams, D. F., \& Eloy, R. (2000) Biomaterial-induced sarcoma: a novel model to study pre-neoplastic change. American Journal of Pathology, 156(4), 1455-1467.

Kommers, G. D., Ilha, M. R. S., \& Barros, C. S. L. (1999). Dioctofimose em cães: 16 casos. Ciência Rural, 29(3), 517-522.

Meuten, D. J. (2017). Tumors in domestic animals. Iowa State Press, (5th Edition).

Moizhess, T. G. (2008) Carcinogenesis induced by foreign bodies. Biochemistry, 73(7), 763-775.

Nambiar, P. R., Jackson, M. L., Ellis, J. A., Chelack, J., Kidney, B. A., \& Haines, D. M. (2001) Immunohistochemical detection of tumor suppressor gene p53 protein in feline vaccine site-associated sarcomas. Veterinary Pathology, 38(2), 236-238.

Norouzi, R., Manochehri, A., \& Hanifi, M. (2017). A case report of human infection with Dioctophyma renale from Iran. Urology Journal, $14,3043-3045$.

Pedrassani, D., Wendt, H., Rennau, E. A., Pereira, S. T., \& Wendt, S. B. T. (2014). Dioctophyme renale (Goeze, 1782) in a cat with a supernumerary kidney. Revista Brasileira de Parasitologia Veterinária, 23(1), 109-117.

Peiffer, R. L., Monticello, T., \& Bouldin, T. W. (1988). Primary ocular sarcomas in the cat. Journal of Small Animal Practice, 29, $105-116$.

Pereira, B. J., Girardelli, G. L., Trivilin, L. O., Lima, V. R., Nunes, L. C., \& Martins, I. V. F. (2006). Ocorrência de dioctofimose em cães do município de Cachoeiro do Itapemirim, Espírito santo, Brasil, no período de maio a dezembro de 2004. Revista Brasileira de Parasitologia Veterinária, $15,123-125$.

Perera, S. C., Rappeti, J. C. S., Milech, V., Braga, F. A., Cavalcanti, G. A. O., Nakasu, C. C., Durante, L., Vives, P., \& Cleff, M. B. (2017). Eliminação de Dioctophyme renale pela urina em canino com dioctofimatose em rim esquerdo e cavidade abdominal - primeiro relato de caso no Rio Grande do Sul. Arquivo Brasileiro de Veterinária e Zootecnia, 69(3), 618-622.

Perlmann, E., Rodarte-almeida, A. C. V., Albuquerque, L., Safatlo, A. M. V., Pigatto, J. A. T., \& Barros, P. S. M. (2011). Feline intraocular sarcoma associated with Phthisis bulbi. Arquivo Brasileiro de Medicina Veterinária e Zootecnia, 63(3), 591-594.

Ranen, E., Lavy, E., Aizenberg, I., Perl, S., \& Harrus, S. (2004). Spirocercosis-associated esophageal sarcomas in dogs. A retrospective study of 17 cases (1997-2003). Veterinary Parasitology, 119(2-3), 209-221.

Rappeti, J. C. S., Mascarenhas, C. S., \& Perera, S. C. (2016). Dioctophyme renale (Nematoda: Enoplida) in domestic dogs and cats in the extreme south of Brazil. Brazilian Journal of Veterinary Parasitology, 26(1), 119-121.

Robbins, S. L., Cotran, R. S., \& Kumar, V. (2015). Neoplasia. Robbins and Cotran's Pathologic Basis of Disease; Elsevier Health Sciences: Makati City, Philippines, (8a ed.) Cap. 7.

Sapin, C. F., Silva-Mariano, L. C., Piovesan, A. D., Fernandes, C. G., Rappeti, J. C., Braga, F. A. V., Cavalcante, G. A., Rosenthal, B. M., \& Grecco, F. B. (2017). Estudo anatomopatológico de rins parasitados por Dioctophyme renale em cães. Acta Scientiae Veterinariae, $45,1-7$. 
Research, Society and Development, v. 10, n. 6, e50410615705, 2021

(CC BY 4.0) | ISSN 2525-3409 | DOI: http://dx.doi.org/10.33448/rsd-v10i6.15705

Silva, C. C., Cavalcanti, G. A. O., Braga, F. V. A., Guim, T. N., Gomes, L. G. S., \& Rappeti, J. C. S. (2017). Dioctophyme renale parasitism in domestic cat in Rio Grande do Sul, Brazil - first reported case Semina: Ciências Agrárias, 38(4), 2215-2219.

Souza, M. S. D., Duarte, G. D., Brito, S. A. P. D., \& Farias, L. A. D. (2019). Dioctophyma renale: Revisão. Pubvet, 13(6), 1-6.

Verocai, G. G., Measures, L. N., Azevedo, F.D., Correia, T. R., Fernandes, J. L., \& Scott, F. B. (2009). Dioctophyma renale (Goeze, 1782) in the abdominalcavity of a domestic cat from Brazil. VeterinaryParasitology, 12(161), 342-344.

Zabott, M. V., Pinto, S. B., Viott, A. M., Tostes, R. A., Bittencourt, L. H. F. B., Konell, A. L., \& Gruchouskei, L. (2012). Ocorrência de Dioctophyma renale em Galictis cuja. Pesquisa Veterinária Brasileira, 32(8), 786-788. 